\title{
Éditorial: The Times They Are A-Changin' (Les Temps Changent)
}

Ce n'est peut-être qu'une coïncidence si tant de changements semblent se produire en 2009. Pour les chercheurs se consacrant à la recherche sur la population âgée du Canada et pour le milieu de la recherche internationale en matière de gérontologie, je crois que la récession mondiale nous remettra en question et que nous reconsidérerons la plupart des hypothèses sur lesquelles nos recherches ont été fondées au cours des dernières décennies. Permettez-moi de vous en mentionner quelques-unes.

Nous avons généralement supposé que la pauvreté chez les personnes âgées baissait parce que les revenus et les paiements de transfert des gouvernements augmentaient. Quelles seront les répercussions du déclin rapide du marché mondial des valeurs mobilières et de l'effondrement des prix de l'immobilier en de nombreux endroits ainsi que leurs effets sur tous les ordres de gouvernement de même que la réaction de ces derniers sur les générations de personnes âgées à venir? $\mathrm{Au}$ lieu que nous analysions les tendances prétendant que la retraite anticipée ou la retraite à l'âge de 65 ans sont de plus en plus la norme, quelles seront les conséquences de la retraite obligatoire, des gens travaillant au-delà de 65 ans et des gens retraités qui ont besoin de retourner travailler parce qu'ils n'ont pas les moyens de vivre de leurs épargnes et de leurs pensions qui, il y a quelques années, étaient suffisantes selon eux pour réaliser leurs aspirations des dernières années? Au lieu d'analyser la tendance à travailler moins d'heures par semaine, aurons-nous besoin de considérer le fait que les aidants naturels travaillent de longues heures et ont moins d'heures pour aider? Au lieu de considérer la tendance vers un plus grand nombre d'années de vie sans incapacité, devrons-nous tenir compte d'un nombre grandissant de personnes âgées qui, en raison du stress causé par ces temps incertains, ont davantage de problèmes de santé physique et mentale? Si ces changements, ainsi que d'autres facteurs, bouleversent les normes sur lesquelles nous avons appuyé nos démarches, quelles seront les répercussions sur le système de santé et de services sociaux que nous avons cherché à améliorer?

En ce moment même, il est difficile de dire si les changements suggérés plus haut de même que plusieurs autres non mentionnés s'effectueront à court ou à long terme. Même à court terme, les défis seront importants pour les personnes âgées. Pour le milieu scientifique, nous avons besoin d'examiner la pertinence d'apporter d'éventuelles modifications aux théories, modèles et méthodes sur lesquels la recherche s'est fondée dans le passé. Peut-être aurons-nous besoin de nouveaux modèles, méthodes et théories pour comprendre et expliquer les changements qui s'opèrent et pour contribuer aux politiques publiques visant la réduction des effets négatifs sur les populations âgées comme les contrecoups de la récession mondiale deviennent de plus en plus manifestes.

Juste au moment où nous souhaitons repenser nos théories et modèles de recherche, La Revue canadienne du vieillissement / Canadian Journal on Aging (RCV/CJA) a mis en œuvre un processus de révision de ses futures stratégies pour améliorer la diffusion de nos efforts de recherche. Dans le présent numéro, nous faisons affaire avec un nouvel éditeur : la Cambridge University Press (CUP). J'ai confiance que ce changement permettra d'améliorer le service offert aux lecteurs et aux auteurs, mais le changement qui m'enthousiasme le plus est que la RCV deviendra une véritable revue internationale. Qu'est-ce que cela signifie réellement pour les auteurs et les lecteurs?

Il n'y a aucun doute que la RCV continuera d'être le principal chef de file de la recherche spécialisée sur la population âgée du Canada effectuée par nos chercheurs les plus doués. Ce qui changera, c'est que le lectorat s'élargira à un auditoire international en raison du réseau planétaire de distribution et de marketing de la CUP. Grâce à une présence internationale de plus grande envergure, le lectorat peut aussi s'attendre à voir davantage d'articles rédigés par les plus grands chercheurs du monde dans les pages de la RCV. Bien qu'il y aura vraisemblablement des obstacles lorsque ces changements surviendront, j'ai confiance que les changements que la RCV apportera avec notre nouvel éditeur renforceront la RCV à mesure que nous irons de l'avant. Oui, "les temps sont en train de changer » et, avec eux, notre recherche et notre journal doivent aussi changer.

Comme toujours, vous pouvez communiquer avec moi à l'adresse mark.rosenberg@queensu.ca si vous avez des commentaires au sujet de cet éditorial ou de tout autre aspect de la RCV.

Mark Rosenberg

Rédacteur en chef 